\title{
COHESIÓN SOCIAL Y GOBERNABILIDAD EN AMERICA LATINA, 2007
}

\section{AUTOR: CORPORACIÓN ESCENARIOS}

COHESIÓN SOCIAL Y GOBERNABILIDAD EN AMÉRICA LATINA, 2007

El presente documento fue preparado por la Corporación Escenarios para el estudio, análisis y discusión del tema Cohesión Social y Gobernabilidad en América Latina desarrollado en el VIII Foro de Biarritz, llevado a cabo durante los días 8 y 9 de octubre de 2007, en Santiago de Chile,

El VIII Foro de Biarritz reunió aproximadamente 200 personalidades de América Latina y Europa, entre quienes se encontraban nueve ex presidentes latinoamericanos, seis alcaldes de ciudades de Ecuador, Perú y Argentina y destacados políticos, académicos y autoridades internacionales.

En representación del Politécnico Granco-lombiano participó el Doctor Pablo Michelsen Niño, Rector de la Institución, quien hizo una disertación sobre educación en el Panel sobre Cohesión Social y Educación; Cultura y Sociedad de la Información.

\section{EL CONCEPTO DE COHESIÓN SOCIAL}

La evolución de las sociedades impone nuevas pautas en sus modelos de administración social.
América Latina no ha sido la excepción a esta regla. Los viejos conceptos individualistas de pobreza que "asociaban el desarrollo y la riqueza a la visión unidimensional del ingreso como único factor"1 (Sarmiento, 2007) y las políticas sociales con la simple oferta de bienes sociales como la salud, la educación y la vivienda, han sido reemplazados por políticas colectivistas que apuntan a la atención integral de necesidades básicas insatisfechas, la formación de nuevas capacidades dentro de un entorno de libertad y el respeto a los derechos humanos como marco de referencia. Esta visión, más compleja del desarrollo social introduce, además, enfoques de demanda que aportan "señales de mercado" que ayudan a mejorar la eficiencia de las políticas sociales, como sería el caso de los programas de focalización.

La cohesión social debe entenderse dentro de este nuevo contexto. Si, como señala el diccionario de la Real Academia de la Lengua, la cohesión es "la cualidad o propiedad de las cosas cuyas partes están fuertemente unidas física o espiritualmente", los ele- 
El concepto de cohesión social en América Latina difiere del europeo; en la primera, el objetivo de alcanzar la cohesión social está directamente relacionado con las posibilidades de superar la desigualdad social. mentos fundamentales de ella son la inclusión a través de la cual se proveen los bienes y servicios que integran materialmente la sociedad y el sentido de pertenencia que integra simbólicamente y le da coherencia a los procesos de organización social y participación comunitaria acercándolos al concepto de nación. Inclusión y sentido de pertenencia son, entonces, los elementos básicos de la ecuación de la cohesión social. Por lo demás, es definitivo pasar, en los términos de Durkheim, de una visión mecánica de la cohesión social a una visión orgánica de la misma, ${ }^{2}$ que incorporaría temas como la innovación, la igualdad, la integración, el desarrollo sustentable y la equidad. ${ }^{3}$

La búsqueda de la cohesión como política de Estado lleva a confundir la acción pública con la protección social de los ingresos y, de manera más general, con la protección contra todos los riesgos, naturales y sociales que pueden amenazar al ciudadano. La cohesión social se debilita cuando este último percibe que el Estado no lo protege de los peligros que lo acechan o que protege más a unos que otros a través del aseguramiento directo, del establecimiento de sistemas contributivos basados en subsidios cruzados entre los estratos superiores o mediante la formación de "comunidades de riesgos" a través de las cuales los propios ciudadanos organizan su propia protección. Asimismo, lo puede hacer a través de redes focalizadas de protección social. Un sistema de protección social es, en última instancia, un acuerdo político al que concurre la sociedad para establecer las bases sobre las que desea construir y regular su convivencia.

En relación con este tema también debe avanzarse en la minimización del "riesgo moral" en el caso de privatizaciones de las empresas de servicios públicos para proteger los intereses más sensibles socialmente. La teoría de la protección social frente al riesgo se convierte así en la mejor política de cohesión social como lo ha señalado la Presidenta de Chile, Michelle Bachelet. A pesar de que la teoría de la capacidad asimétrica de respuesta ante los riesgos modernos, planteada por UlricK Beck en Alemania, se aplicaría, de manera preferente, a las sociedades desarrolladas, en América Latina el problema es mucho más complejo en la medida en que se trata de formas de exclusión estructural de miles de personas sin voz en los términos de Hirschman. 4

El concepto de cohesión social en América Latina difiere del europeo; en la primera, el objetivo de alcanzar la cohesión social está directamente relacionado con las posibilidades de superar la desigualdad social. Esto es evidente si se tiene en cuenta que se trata de la zona del mundo donde existen los mayores niveles de desigualdad relativa como lo prueba el hecho de que hoy ningún país latinoamericano presente niveles de desigualdad inferiores a los que tenía hace tres décadas 5 (Ocampo en BID, 2004).

1. SARMIENTO, Alfredo. "La Inclusión: Marco Conceptual” Documento en borrador, marzo 2007.

2. VEUNAC, Michel, Teniente Alcalde de Biarritz. Discusión del documento sobre Cohesión Social en el V Encuentro del Grupo de Biarritz, realizado en ciudad de Guatemala, 20 y 21 de julio de 2007.

3. IBARRETXE, Juan José, Lehendakari del País Vasco. Discusión del documento. V Encuentro del Grupo de Biarritz, Guatemala, 20 y 21 de 2007.

4. Comentarios del señor Horst Grebe, al documento sobre Cohesión Social presentado en el V Encuentro del Grupo de Biarritz, Guatemala, 20 y 21 de 2007.

5. Banco Interamericano de Desarrollo. "Inclusión Social y Desarrollo Económico en América Latina," 2004. Página 39. 
El mapa de la exclusión social reciente en América Latina muestra dos fenómenos bastante paradójicos. De una parte, es claro que el aumento de la inversión social como parte del producto interno bruto, que pasó, durante la pasada década, del 10.4\% al $13.1 \%$ (inferior a la de Europa que esta cercana al $30 \%$ ) indica un esfuerzo formidable en la búsqueda de mejores condiciones de equidad. De otro lado, aparecen síntomas de retroceso o empobrecimiento como los altos índices de desempleo abierto que han crecido durante la última década y la propia informalización de la economía que deterioró la calidad de los mercados de trabajo caracterizados en la región por la falta de estímulos y políticas para el uso reproductivo del excedente. ${ }^{6}$ Se trata de un panorama variopinto donde aparecen luces como el aumento en la expectativa de vida, la disminución del analfabetismo, la masificación de la enseñanza primaria, la disminución de las tasas de fecundidad y la incorporación de la mujer al mercado de trabajo que alternan con sombras como el empobrecimiento, el crecimiento de la informalidad y el agravamiento de las condiciones generales de gobernabilidad.

La visión europea de la cohesión social se distancia del concepto material de la desigualdad social latinoamericana para relacionarse más cercanamente con el de sentido de pertenencia, la participación política, la integración social y el desarrollo como remoción de las barreras que impiden la libertad en los términos de Amartya Sen (2000). Se trata, por decirlo de alguna manera, de una visión multidimensional de la pobreza. El asunto de la convivencia dentro de la diversi- dad étnica existente en los países que forman parte de la Unión Europea ampliada tiene, por supuesto, una mayor importancia que la que se le confiere en América Latina donde existen fuertes raíces que permiten hablar de una identidad latinoamericana. Tuvieron que suceder cientos de conflictos y no pocas guerras para llegar al concepto de cohesión social asociado con los tres postulados capitulares de la Revolución Francesa: la fraternidad, como sentido de pertenencia a una comunidad, la igualdad, como parte efectiva de la misma, y la equidad, que representa los vínculos sociales entre la ciudadanía.

Desde el año 2000 todos los países europeos están realizando Planes Nacionales de Lucha contra la Exclusión. En el año 1990 se creó el Observatorio de Políticas Nacionales Contra la Exclusión Social que se ocupa de hacerle el seguimiento a temas como el empleo, acceso a recursos, prevención de riesgos de exclusión, acciones para ayudar a los más vulnerables y movilización de los agentes relevantes. Desde Lisboa, se incluyó el término de "cohesión social" en el léxico europeo que, aparentemente, parecía más comprensivo que el de inclusión social.

\section{LA INCLUSIÓN COMO PARTE DE LA COHESIÓN SOCIAL}

El término "exclusión", contrapuesto al de inclusión, fue acuñado en Francia en el año de 1974 para referirse a los ciudadanos franceses no beneficiarios del sistema de seguridad social. La exclusión tenía que ver entonces con la incapacidad del individuo para participar en el funcionamiento básico, político y social de la sociedad en que vive. (Tsakloglor y Papadopulos, en BID, 2004). A través de la inclusión, según los expertos,

6. Grebe, Horst. Discusión del documento sobre Cohesión Social en el V Encuentro del Grupo de Biarritz, Guatemala, 20 y 21 de julio de 2007. 
se garantizaba el acceso de sectores marginados a procesos institucionales de atención social, se aseguraba el mejoramiento de sus ingresos y se avanzaba en un mejor reparto de los activos productivos.

El paso del concepto de pobreza al de exclusión permite ver el problema de la marginalidad latinoamericana no solamente en términos de existencia de necesidades como de falta de oportunidades y capacidades para poder conseguirlas. La exclusión responde así a realidades dinámicas donde las fuerzas que propenden por la concentración económica terminan por hacer más graves las condiciones mismas de la desigualdad; en la medida en que esta última está relacionada con los ingresos y el acceso a los activos productivos, las medidas para fortalecer la cohesión social a través de una mayor inclusión deben tener en cuenta el acceso de los excluidos a activos productivos hoy excesivamente concentrados como la capacitación técnica, el microcrédito y la tierra. Sobre esta realidad dinámica de la exclusión también se ha dicho que la pobreza reproduce la exclusión en la medida en que bajos niveles de educación, propios de los pobres, conllevan menores posibilidades de empleos calificados. Por ello, no es coincidencial que los estudios existentes sobre la exclusión se han preocupado por abordarla relacionándola con las condiciones generales de marginalidad social y económica mientras que otros lo hacen a través de la identificación de aquellos sectores de la sociedad más afectados por ella. Al primer enfoque lo denominaremos "enfoque de exclusión social" y al segundo, "enfoque de discriminación".

La exclusión social alude a las condiciones generales de desigualdad de una sociedad que impiden el acceso de todos sus ciudadanos a los bienes y servicios que requieren para su supervivencia; como lo ha señalado reiteradamente el BID, las condiciones generales del ingreso son un obstáculo para que estos grupos accedan a los empleos, activos y oportunidades que requieren para superar la pobreza estructural o permanente que es en lo que consiste la transmisión intergeneracional de la desventaja(BID, 2004). En este sentido, el estado del cumplimiento de las metas del milenio como la posible creación de un Observatorio de Exclusión similar al creado en Europa hace varios años, ayudaría muchísimo en la tarea de tomarle de manera permanente el pulso al progreso de la inclusión como fuente de cohesión social.

La discriminación plantea una visión más selectiva del tema de la exclusión pues resulta, según el Tribunal Europeo de Justicia, cuando se aplican reglas diferentes a situaciones comparables o las mismas reglas a situaciones diferentes (BID, 2004). La discriminación estigmatiza y divide la sociedad debilitando sus posibilidades de inclusión. Las políticas de Naciones Unidas han optado por un enfoque mixto entre exclusión y discriminación a través de la celebración de cumbres especializadas sobre grupos marginados como la de Beijing sobre la mujer (1995) y cumbres generales como la Cumbre Social del Milenio (1996). El Banco Interamericano trabaja el tema a partir de los sectores excluidos, más concretamente, los afrodescendientes, los pueblos indígenas, los discapacitados y los enfermos de SIDA.

Las mujeres estarían localizadas en una categoría especial como parte de "formas 
complejas de discriminación", por ejemplo mujeres negras desempleadas y, por supuesto, pobres. La incorporación masiva de las mujeres al mercado de trabajo, como consecuencia de su ingreso al sistema educativo, hace varias décadas, ha sido señalada como uno de los fenómenos sociales más importantes en la región en la medida en que afectó estructuras económicas, sociales, culturales y familiares causantes de fenómenos como el subempleo de las mujeres jóvenes. América Latina tiene mucho que aprender de los aciertos de las políticas afirmativas en pro de la mujer del Norte de Europa y de los errores de las políticas discriminantes de la Europa mediterránea donde aun existe una fuerte discriminación de género resultante de conocidas tradiciones machistas, autoritarias y conservadoras.

Los países latinoamericanos han consagrado en sus constituciones normas que garantizan la protección formal de distintos grupos minoritarios y han expedido leyes que consagran políticas de acción afirmativas como el establecimiento de cuotas para garantizar espacios a la participación de género. Sin embargo, este concepto de exclusión por discriminación no comprende otros grupos sociales, religiosos, sexuales, migrantes o étnicos que esperan del Estado más acciones de protección que de apoyo.

Los migrantes latinoamericanos podrían ser considerados, especialmente los que viven en Europa y Estados Unidos, como uno de los grupos que requieren mayor atención por la discriminación a la que se encuentran sometidos. La política migratoria de la Unión Europea actual es claramente discriminatoria contra los tra- bajadores inmigrantes a los cuales, hasta la década de los ochenta, se les daba un tratamiento de simple acogida. Los extranjeros representan hoy el 5.4\% de la población europea (Real Instituto Elcano, 2006); la mitad de ellos llegan a España, Italia y Portugal; de los veinticinco millones de latinoamericanos que emigran anualmente el 12\% se dirige hacia Europa. El 4\% de los latinoamericanos viven por fuera de sus países siendo los casos más notables el de los salvadoreños (14.7\%) y el de los mexicanos (9.4\%) (Real Instituto Elcano, 2006). Los países con mayor número de inmigrantes a Europa eran para el año 2004 Ecuador con 390.000, Colombia con 244.000, Argentina con 109.000, Perú con 55.000 y República Dominicana con 44.000. (Ver mapa No. 1).

La actual política migratoria europea no solo exime a la Unión Europea de proteger a los migrantes contra la discriminación, sino que sus mismas disposiciones suelen ser causa de su discriminación. (Melis en BID, 2004). Los migrantes son sometidos a formas de exclusión pasiva en las comunidades donde llegan o de exclusión activa a través de leyes segregantes. En algunos países, como la Gran Bretaña, son tolerados pero no aceptados. El racismo, la xenofobia y la utilización política del fenómeno migratorio han acentuado las políticas discriminantes contra los latinoamericanos en Europa. Castells distingue los modelos de "exclusión diferencial," (Alemania) donde los trabajadores migrantes son considerados como "invitados", los modelos de asimilación (Francia), el Estado trabajando para incorporar a los individuos como iguales) y los modelos "pluralistas o multiculturales” (BID, 2004). 
El tratamiento

de los

migrantes debe

partir del hecho

de que todos

los miembros

de una

sociedad deben

pertenecer a

su comunidad

política; la

exclusión de

los migrantes

de esta

comunidad

política, de la

sociedad o de

las dos desafía

la aspiración europea de gobernaren democracia.
El tratamiento de los migrantes debe partir del hecho de que todos los miembros de una sociedad deben pertenecer a su comunidad política; la exclusión de los migrantes de esta comunidad política, de la sociedad o de las dos desafía la aspiración europea de gobernar en democracia. Así como en el siglo XIX se diferenciaban las elecciones "administrativas" locales de las elecciones "políticas" nacionales, así también deberían hoy diferenciarse las condiciones de participación de los migrantes para reconocer la figura de la "doble nacionalidad" que les permite adquirir una carta de ciudadanía extranjera sin renunciar a la de nacimiento.

La promoción de la inclusión social en América Latina es la mejor manera de prevenir la exclusión de los migrantes latinoamericanos en Europa o en cualquier parte del mundo mientras avanzan otras iniciativas como la posibilidad de hacer convenios con los países de origen para administrar la inmigración, una política de asilo común y un Fondo Europeo para los Refugiados para financiar su recepción, integración y repatriación; se abre camino de manera, definitiva, la noción de una ciudadanía transnacional donde todos podamos ser considerados "ciudadanos del mundo".

Los afrodescendientes representan el $30 \%$ de la población latinoamericana, aproximadamente ciento cincuenta millones de personas, la mitad de ellas en Brasil y se localizan en los estratos más bajos en la distribución de ingreso con el mayor número de necesidades básicas insatisfechas. Provienen de los africanos esclavizados en América Latina, en algunos países el término se confunde con el de población negra y en otros se les asocia directamente con el país de residencia, por ejemplo, los afroecuatorianos. (RANGEL, 2006) ${ }^{7}$ A pesar de que, lamentablemente, la Declaración del Milenio no se refiere precisamente a este grupo, los estudios han demostrado que su condición de raza en la mayoría de los países coincide con los sectores de población más pobres y marginados social y geográficamente.

Los indígenas: La población indígena en América Latina, según los últimos censos supera los 30 millones de personas. El peso de ella, eminentemente rural, es fundamental en algunos países como Bolivia (62.2\%), Guatemala (41\%), Perú (32\%), Ecuador (6.8\%) y México (6.4\%) (ver mapa $\mathrm{N}^{\circ} .2$ ). Aunque casi todas las constituciones de la región reconocen el carácter multiétnico de sus sociedades y la mayoría de sus gobiernos han firmado la Convención 169 de la OIT, que defiende la diversidad de los pueblos indígenas del mundo, los indígenas latinoamericanos siguen formando parte de los núcleos de población más discriminados en la región, que mantienen herencias coloniales de exclusión cuando la palabra "indio" se utilizaba como criterio discriminante frente a los conquistadores blancos. Los indígenas latinoamericanos son "los excluidos de los excluidos”; estudios recientes señalan, por ejemplo, que si dicha población pudiera alcanzar la productividad promedio en cada uno de los países donde habitan, la economía de Bolivia crecería en un 36.7\% y la de Guatemala en 13.6\%. (CEPAL, AECI, SEGIB, 2007); la tasa de pobreza de estos grupos aborígenes en algunos países, como Guatemala, es del 77\%

7. RANGEL, Martha. Documento CEPAL, Pueblos indígenas y afrodescendientes de América Latina y el Caribe: información sociodemográfica para políticas y programas, mayo 2006. 
frente al promedio nacional del 44\% (CEPAL, AECI, SEGIB, 2007). La mortalidad de los niños indígenas latinoamericanos es superior en un $60 \%$ al del resto de infantes.

Esta situación de desprotección secular talvez explica porque los indígenas que habitaban estas tierras se consideraban a sí mismos como "seres humanos" para diferenciarse de los "seres extraños" como se referían a quienes venían a colonizarlos; su mayor preocupación no era, ciertamente, “vivir mejor" sino "vivir bien.” La campesinización de los indígenas, que confunde la protección de su identidad con la defensa de sus tierras, ha terminado por convertir la protección de estas comunidades nativas en un problema de reparto de parcelas y apoyos para trabajarlas.

Los discapacitados constituyen otro importante sector discriminado. En Chile, representan el $4.3 \%$ de la población total y en Ecuador el 13.2\%. (BID, 2004). Hasta el año de 1950 la discapacidad física, mental o sensorial fue considerada como "otredad" (Massiah en BID, 2004); en los años setenta se registró un avance notable cuando se le quitó a la discapacidad la condición peyorativa de "deficiencia" que llevaba a concentrarlos como "minusválidos" en guetos como escuelas especiales, clínicas cerradas y centros de rehabilitación focalizados. A partir de entonces, los gobiernos, nacionales y locales, comenzaron a adecuar sus infraestructuras sociales tradicionales para garantizar su convivencia con el resto de ciudadanos; nació el interés por adecuar las ciudades y los espacios políticos a las particulares circunstancias de incapacidad de estos ciudadanos.
El SENTIOO de PERTENENCIA COMO FUENTE DE COHESIÓN SOCIAL

La cohesión social también tiene que ver con la identidad y la libertad entendida como la capacidad de una persona para adelantar acciones respecto a asuntos que tiene razones para valorar. (Sen, 2000); libertad y necesidad gobiernan el funcionamiento de instrumentos previstos para satisfacer necesidades como el Estado, el mercado, el sistema jurídico y de formulación de políticas sociales inspiradas en valores y derechos humanos. Esta búsqueda de la satisfacción de la necesidad a través de la libertad excluye, por supuesto, la apelación emocional a motivaciones irracionales como el populismo o el fundamentalismo religioso (Sen, 2000). Para la sabiduría Maya la cohesión social es base de la armonía y esta de la identidad y la sustentabilidad, ${ }^{8}$ lo cual explica porqué el diálogo intercultural es el mejor instrumento para abordar el viejo dilema igualdad social y diversidad cultural. ${ }^{9}$

El conjunto de relaciones sociales cercanas como las que nacen de la familia, el vecindario, las asociaciones de padres o los vecindarios, sumadas alas quellevan a compartir sentimientos degrupo por razones de etnia, raza o religión y las que identifican los individuos dentro de una misma clase, conforman el "capital social" de una sociedad. Este capital puede ser positivo, si añade valor al conjunto social, aumentando por ejemplo, la solidaridad o negativo si conlleva situaciones de ruptura como las que resultan de los nexos, contactos y redes entre organizaciones criminales. Como patrimonio simbólico de la sociedad en términos de manejo de normas, redesylazos sociales de confianza que permiten

8. COLOM, Álvaro, Candidato a la Presidencia de Guatemala. Discusión del documento en el evento mencionado antes.

9. GREBE, Horst, Ex Ministro de Economía de Bolivia. Discusión del documento sobre Cohesión Social en el V Encuentro del Grupo de Biarritz, Guatemala, 20 y 21 de julio de 2007. 
reforzar la acción colectiva ${ }^{10}$ (Ibáñez, 2002), el capital social, así entendido, se convierte así en la más tangible expresión de la cohesión social.

Un reciente Informe sobre el Desarrollo Humano en América Latina se concentró, específicamente, en este tema de la identidad como fuente de cohesión social. ${ }^{11}$ (PNUD, 2006); el estudio encontró una fuerte carga de pesimismo en los jóvenes latinoamericanos que sienten que sus condiciones de vida son peores que las de sus padres, así sean, como en efecto lo son, mejores en la realidad. Los entrevistados reforzaron la vieja idea, prevaleciente en la región, de concebir la identidad como "la negación del otro": la gente, en América Latina, se identifica diferenciándose de los demás. Dicho patrón de identificación negativa produce fragmentación social y exclusión; con razón se ha dicho, al hablar del identidad cultural latinoamericana, que su principal problema no es el individualismo, sino la cultura individualista que la caracteriza ${ }^{12}$ (Ibáñez, 2002). Es preciso, entonces, construir nuevos patrones de identidad positiva para afianzar un nuevo sentido regional de la cohesión social; no es una tarea fácil en la medida en que hemos trabajado tanto en establecer nuestras diferencias que ya no sabemos ni qué nos une ${ }^{13}$, y que hemos venido liquidando la autoes- tima hasta caer en una especie de síndrome del menosprecio. ${ }^{14}$

El "ser latinoamericano", sin embargo, existe: está allí presente, en todo lo que sentimos, pensamos, soñamos, vivimos. América Latina, a pesar de haber sido conquistada antes que descubierta, esclavizada antes que reconocida y reglamentada antes que comprendida $^{15}$ (Massur, citado por Houllebecq, 2000), puede considerarse como una fragua étnica donde se han mezclado y confundido distintas manifestaciones étnicas y culturales sin producir los conflictos y las guerras que han sido de común ocurrencia en la convulsionada historia de Europa. El mestizaje, la capacidad de mezclarnos sin enfrentarnos, ha sido, quizás, el rasgo más sobresaliente de la cultura latinoamericana; la tendencia hacia el multiculturalismo que expresa esta forma de convivencia tiene que ver con esa capacidad de concatenar distintas expresiones culturales, especialmente étnicas, a través de hibridaciones sucesivas que van produciendo síntesis afortunadas de nuevas identidades ${ }^{16}$ (García Canclini, 2006). En esa fragua étnica se "'funden" las diferencias raciales como sucedió entre indios y colonizadores, criollos y afroamericanos y entre los propios europeos colonizadores provenientes de distintas etnias, regiones y países. ${ }^{17}$ (Piñón, 2006 ).

10. IBAÑEZ, Ana María, LINDERT, Kathy y WOOLCOCK, Michael. "Social Capital in Guatemala: A Mixed Methods Análisis,” A background paper for the Guatemala Poverty Assessment (GUAPA), junio 26 de 2002.

11. PNUD. "Informe sobre Desarrollo Humano 2006: Más allá de la escasez: poder, pobreza y crisis mundial del agua," Nueva Cork, 2006.

12. IBAÑEZ, Ana María, LINDERT, Kathy, WOOLCOCK, Michael. Op. Cit.

13. MESA, Carlos, Ex Presidente de Bolivia. Discusión del documento en el evento mencionado antes.

14. ARAYA, Rolando. Discusión del documento en el evento mencionado antes.

15. HOULLEBECQ, Michel (2000), El Mundo como Supermercado, Barcelona: Editorial Anagrama, Colección Argumentos.

16. GARCÍA CANCLINI, Néstor. Departamento de Antropología, Universidad Autónoma Metropolitana. Presentación realizada en el marco del Seminario Preparatorio sobre Industrias Culturales, auspiciado por la Organización de Estados Iberoamericanos. México D.F., Marzo 4, 2006.

17. PIÑÓN, Francisco. "Los Intelectuales, Raíces y Valores Frente al Debate de la Identidad," documento de debate preparado para el 7o Foro de Biarritz por la Organización de Estados Iberoamericanos, octubre 2006. 
La identidad latinoamericana, empero, se encuentra hoy amenazada por un proceso desordenado de informatización que consigue que vaya mas rápido la "integración simbólica" a través de los medios de comunicación que la "integración material," a través de la inclusión social, creando esa sensación de "estar siempre atrás" que crea insatisfacción y expectativas frustradas especialmente en los sectores jóvenes. ${ }^{18}$ (Hopenhayn, 2003). Está establecido, por ejemplo, que los niveles de comunicación a través de Internet que hoy sostienen distintas etnias indígenas desde México hasta la Patagonia contrasta con su exclusión de las sociedades donde cada una de estas comunidades habita.

\section{¿QUUE SE PUEDE HACER HACLAADELANTE?}

Sin pretender caer en la utopía de una "identidad única" que contradice la multiculturalidad propia de la región, sí es posible encontrar raíces muy fuertes sobre las cuales se puede edificar hacia el futuro un sentido más sólido de pertenencia latinoamericana. El español, convertido hoy en una de las tres lenguas más habladas del mundo al comenzar el siglo XXI, es uno de estos comunes denominadores de nacionalidad. Como articulador de la identidad hispánica de los cuarenta millones de hispánicos residentes en los Estados Unidos, el idioma de Cervantes está librando una batalla épica, como las del Cid Campeador, contra quienes, a través de la campaña "English Only", se oponen al fenómeno, imparable, del bilingüismo como patrón de la nueva biculturalidad norteamericana. Los hispánicos no están solos, la lengua castellana es de lejos la más utilizada y la preferida por un millón de norteamericanos que la están estudiando. Y aunque existen signos preocupantes sobre su futuro por las campañas en su contra y el abandono de su uso por parte de la segunda y tercera generación de los propios hispánicos nacidos en los Estados Unidos, el auge de publicaciones, canciones y películas en español, su difusión a través de una cadena de más de 500 emisoras de radio y la importancia, como calificación laboral, que está adquiriendo el bilingüismo inglés- español, permiten hacer un balance favorable sobre su futuro en el país de Lincoln (Corporación Escenarios, 2006).

La comunidad católica latinoamericana es la más numerosa del planeta y una de las más importantes, como lo prueban los aportes del episcopado latinoamericano a la doctrina social de la Iglesia a través de debates históricos como el de la Teología de la Liberación en los años 60 o contra el modelo neoliberal en los 90 . El derecho a la identidad, a "ser diferentes", como parte de una nueva cultura de los derechos humanos que se ha entronizado en la región, presupone la capacidad de cada individuo para elegir las doctrinas religiosas en las cuales cree, teniendo siempre presente que dicha pertenencia se construye con más equidad y una mayor aceptación de la diversidad ${ }^{19}$ (Sen, 2000). El aparecimiento de sectas protestantes en la región ha desafiado la presencia tradicional de la Iglesia Católica, pero también ha reabierto debates y cuestionamientos a sus posiciones doctrinarias que podrían plantear el resurgimiento de un sentimiento

18. Hopenhayn, Martín. "Educación, comunicación y cultura en la sociedad de la información, una perspectiva latinoamericana," CEPAL, Santiago de Chile, 2003.

19. SEN, Amartya. Desarrollo y Libertad. Barcelona: Editorial Planeta, mayo 2000. Pág 272. 
católico que sigue formando parte estructural de la identidad latinoamericana.

Como quedó establecido en el Encuentro de Biarritz del año 2006 en Sao Paulo, el movimientismo social forma parte de esa búsqueda incesante del ser latinoamericano, de esa "latinoamericanidad" entendida, no como una simple sumatoria mecánica de los nacionalismos de países sino como la expresión de una auténtica nacionalidad regional a partir de la cual se podría pensar en un nuevo proyecto de región basado en la cohesión social. Los movimientos sociales en América Latina, como expresión de una nueva dinámica política como los movimientos agrarios y los más recientes de lucha por el agua, han nacido como proyectos de lucha y han terminado convertidos en proyectos de identidad y en algunos casos, como en Bolivia, en autenticas propuestas partidistas. La protesta social y los movimientos que la encarnan no son nuevos en la región. A lo largo de su historia, los movimientos han jugado un papel fundamental en la determinación de la dinámica política latinoamericana; a través de esa especie de dialéctica del disentir de la que habla Giovanni Sartori (2001), han terminado por convertirse en aglutinantes de identidad y fuente de organización política que actúan como grupos en sí que pretenden convertirse en grupos para sí (Poulantzas, 1970).

Desde el grito de independencia de Tupac Amaru que podría considerarse su antecedente más significativo, estos movimientos latinoamericanos han desarrollado esos "vínculos que se sienten" como una forma legítima de construir sociedad y acumular identidad. Por esta razón no es de extrañar que el "movimientismo" siga ocupando hoy un lugar privilegiado en el escenario político latinoamericano donde la gobernabilidad ha quedado supeditada a la posibilidad de que el mensaje de resistencia que estos movimientos expresan se convierta en un proyecto constructivo de identidad y acción política (Cardoso, 2006); como manifestaciones sociales estos colectivos pueden llegar a convertirse en un factor de gobernabilidad en la forma y medida en que sus propuestas, planteadas desde la base, puedan ser asimiladas directamente por los partidos o a través de alianzas de cooptación a través de nuevos frentes políticos sintonizados con la realidad que de alguna manera les dio origen.

La búsqueda de una nueva identidad que fue tema fundamental del Foro de Biarritz 2006, debe trascender el ámbito estrictamente nacional y llegar, como lo propuso el Foro de Biarritz 2005, hasta los espacios locales donde se están creando las nuevas expresiones culturales del futuro; este examen debe incluir todos los actores, públicos y privados, creadores de globalidad, como la sociedad civil y los agentes económicos. La responsabilidad social de estos últimos, en relación con el tema de la identidad y otras materias de la nueva agenda global, como la cohesión social, serán exploradas, precisamente, en el Foro de Biarritz 2007 que se celebra en la ciudad de Santiago de Chile en octubre de este año.

Los gobiernos locales tienen posibilidades limitadas, pero efectivas para fortalecer los tejidos territoriales de cohesión social a través de acciones concretas relacionadas con la inclusión social y la cultura. El enfoque de territorio permite respuestas más eficaces que las nacionales a las múltiples desventajas que enfrentan los más pobres 
en áreas como la infraestructura básica, el acceso a servicios de educación y salud, nuevas oportunidades de generación de empleos e ingresos y participación social. En zonas especialmente apartadas esta condición puede ser particularmente útil para "focalizar" la atención del Estado. ${ }^{20}$ La sostenibilidad de estos programas locales de inclusión debe tener en consideración aspectos financieros, externos, técnicos, sociales y ambientales. ${ }^{21}$

\section{GOBERNABILIDAD Y COHESIÓN SOCIAL}

El término "gobernabilidad" fue utilizado por primera vez en 1975 en el informe de la Comisión Trilateral conformada por Michael Crozier, Samuel Huntington y Joji Wakanuti quienes entonces la definieron como "la capacidad de respuesta de las instituciones gubernamentales a las demandas $\mathrm{y}$ aspiraciones que surgen de la sociedad civil." Los expertos mencionaron entonces los peligros del "exceso de democracia" y destacaron, como elementos relevantes para su análisis, la erosión de la autoridad por la incapacidad estatal para atender todas las demandas sociales por parte del Estado- bienestar y la sobrecarga del gobierno como consecuencia de la intensificación de la competencia política.

En América Latina, la teoría de la gobernabilidad nació como una teoría de la ingobernabilidad y fue utilizada, inicialmente, para referirse a los procesos de transición democrática que estaban teniendo lugar en el área y las circunstancias que harían posible la transición de los regímenes autoritarios militares a las nuevas democracias. Posteriormente se utilizó de una manera más instrumental para calificar los procesos y normas que permitirían el funcionamiento de las administraciones públicas y después, durante los años 90, para referirse a "la capacidad que tienen los gobiernos elegidos democráticamente para dar órdenes que responden a demandas sociales y la aceptación de estas últimas por parte de los gobernados."

Cuando aludimos a esta capacidad para "dar órdenes" que nace, formalmente, del sistema de representación política popular, nos estamos refiriendo a la "gobernabilidad democrática"; en los regímenes autoritarios también existe "gobernabilidad”, se gobierna, sí, pero el origen de ésta autoridad y su ejercicio no son legítimos. Dicha legitimidad, que va de la mano con la gobernabilidad, resulta del origen de los poderes, de la aplicación de los mismos y de la aceptación por los ciudadanos de las reglas de juego establecidas; puede ser institucional o social y es funcional a la forma de delegación de poderes como lo plantearon en su momento Rousseau en un plano teórico y Simón Bolívar en otro eminentemente práctico.

La legitimidad formal tiene que ver con el sistema político y la forma de elección y actuación de los partidos políticos, con la independencia del poder electoral y con las instituciones que conforman la división de poderes en un juego de pesos y contrapesos de poder en los términos descritos por Montesquieu cuando habla de la necesidad, en cualquier sistema representativo, de que "el poder frene el poder". Este último se legitima a través de su ejercicio de manera transparente y justa, que es en lo que consiste el llamado "buen gobierno" que involucra,

20. VI Foro de Biarritz, Bogotá, Colombia, 2005.

21. GARZÓN, Luís Eduardo. VI Foro de Biarritz, Bogotá, Colombia, 2005. 
La meta principal es, sin embargo, la búsqueda de una mayor legitimidad social por medio de políticas que fortalezcan la cohesión social mediante la inclusión y el fortalecimiento del sentido de pertenencia. según el Fondo Monetario Internacional y el Banco Mundial, aspectos como la gestión del sector público, rendición de cuentas, información, transparencia y un marco legal confiable orientado a buenos fines y con posibilidades de permanente refrendación a través de figuras como iniciativas ciudadanas y acciones populares que convierten la democracia, en los términos de Renan, en un plebiscito diario.

La legitimidad social resulta así de la forma como el gobierno atiende las demandas sociales de los ciudadanos y de la propia percepción que ellos tienen sobre su voluntad y capacidad para hacerlo. Los déficit democráticos generalmente van acompañados de déficit sociales. Está demostrado que la democracia sustentada en la pobreza y la desigualdad no garantiza la construcción de ciudadanía, requisito básico de la gobernabilidad; dicho de otra forma: tenemos ciudadanos políticos, pero no tenemos ciudadanos sociales. ${ }^{22}$ No es la privación material, sino la falta de cohesión social la que determina las diferencias sociales. ${ }^{23}$

La crisis reciente de gobernabilidad en América Latina se asocia a la crisis de un modelo injusto de desarrollo que no aumentó el crecimiento ni mejoró las condiciones de equidad, a la incapacidad de los sistemas políticos de la región para responder a exigencias sociales que tuvieron que ser canalizadas de manera contestataria en lo que se ha conocido como la democracia callejera y a la propagación, en la región, de nuevas patologías globales como el narco- tráfico, la corrupción, el armamentismo y el terrorismo.

El objetivo de la nueva red de gobernabilidad latinoamericana debe ser el fortalecimiento de la legitimidad formal a través de una redefinición de roles de actores tradicionales como el Estado, la sociedad civil, los agentes económicos, los partidos políticos, las instituciones públicas, los movimientos sociales y las regiones, y el establecimiento de nuevas formas de relación entre ellos, a través de procesos como la concertación, la descentralización, la integración y la participación. La meta principal es, sin embargo, la búsqueda de una mayor legitimidad social por medio de políticas que fortalezcan la cohesión social mediante la inclusión y el fortalecimiento del sentido de pertenencia. La creación de una nueva institucionalidad social parece vital en el camino de restablecer la gobernabilidad social en la región a partir del reconocimiento de que la pobreza tiende a enfrentar al ciudadano con el Estado y de que este no es débil solamente por su tamaño, sino porque no está bien relacionado con la ciudadanía. ${ }^{24}$

\section{POLIIIICAS DE INCLUSIÓN YMEDIDAS INCLUSIUAS PARA LA COHESIÓN} SOCIAL

Las políticas inclusivas apuntan a reducir la discriminación en términos genéricos a través del desarrollo de capacidades, el mejoramiento de las condiciones generales del ingreso, la constitución de fondos de cohesión como los europeos que reducen las disparidades sociales y geográficas y la

22. RAMÍREZ OCAMPO, Augusto, Ex Canciller de la República de Colombia. Discusión del documento en el evento mencionado antes.

23. CEREZO B., Marco Vinicio, Director General Fundación para el Ecodesarrollo y la Conservación FUNDAECO. Discusión del documento en el evento mencionado antes.

24. MESA, Carlos, Ex Presidente de Bolivia. Discusión del documento en el evento mencionado antes. 
actualización de políticas tradicionales en materia de empleo, educación e integración de los sectores informales; también operan de manera selectiva integrando a la sociedad sectores marginados como las mujeres, los afrodescendientes, los indígenas, los enfermos de sida, los discapacitados y los núcleos de pobres demostrados. La inclusión debe contribuir a fortalecer el concepto de solidaridad como forma de integración positiva mediante el desarrollo de formas asociativas a través de las cuales se pueda expresar la sociedad civil. La responsabilidad social empresarial está incluida en este catálogo de políticas solidarias para la cohesión social. Las políticas relacionadas con el sentido de pertenencia, como parte de una estrategia amplia de cohesión social, apuntan al fortalecimiento del capital social a través de campañas educativas, programas de gestión cultural basados en la transmisión de valores, ampliación de espacios participativos y programas que fortalecen la legitimidad institucional.

Entre la legitimidad social que se consigue con las estrategias de inclusión y se fortalece con del desarrollo del sentido de pertenencia aparece un tercer tipo de estrategias, las participativas que incluyen la modernización de los partidos políticos, el desarrollo de espacios institucionales para la participación ciudadana y la cooptación institucional de los movimientos contestatarios. Para los efectos del Foro de Biarritz 2007, nuestras propuestas se circunscribirán a las políticas y medidas relacionadas con la inclusión social, el sentido de pertenencia y la responsabilidad social empresa- rial como parte de una gran propuesta de cohesión social.

\section{POLIIIICAS MACROECONÓMICAS PARA LA COHESIÓN SOCIAL}

Las políticas macroeconómicas pueden generar exclusión o inclusión social según produzcan mayores o menores condiciones de equidad y contribuyan, en las épocas de crisis, a reducir de manera contra cíclica los costos sociales de decisiones económicas regresivas. Aunque la opinión latinoamericana ya aceptó que no es posible hacer desarrollo a costa de la estabilidad macroeconómica, todavía queda un largo trecho por andar en el camino de asegurar que las políticas económicas no vayan en contravía del desarrollo social y asegurar así que la equidad quede en el centro de la agenda regional para el desarrollo. Distintos analistas coinciden, por ejemplo, en la necesidad de llevar adelante una profunda reforma fiscal, a partir de un nuevo pacto fiscal, para aumentar la participación pública, redistribuir las cargas territoriales y mejorar las condiciones de progresividad de la estructura tributaria considerada hoy como una de las más regresivas en el mundo. El papel que, dentro de este contexto se asigne al mercado, al Estado y a los agentes económicos, afectará positiva o negativamente las posibilidades de avanzar en materia de cohesión social. ${ }^{25}$

Durante muchos años los análisis sobre la pobreza en América Latina se concentraban en el examen de las diferencias en materia de rentas entre distintos sectores de la población. Hoy sabemos que no necesariamente dos familias que tengan la

25. QUENAN, Carlos, Instituto de Altos Estudios de América Latina en París- IHEAL; MALAMUD, Carlos, Investigador Principal Real Instituto Elcano; GREBE, Horst, Ex Ministro de Desarrollo de Bolivia. Discusión del documento sobre Cohesión Social en el V Encuentro del Grupo de Biarritz, Guatemala, 20 y 21 de julio de 2007. 
misma renta tienen las mismas condiciones de bienestar porque la diversidad humana hace que dos personas con rentas iguales puedan tener distinta capacidad u oportunidad de disfrutarlas (Sen, 2000). También quedó demostrado que los mecanismos de mercado no son los más adecuados para el propósito de reducir las desigualdades sociales y que deben ser complementados con la creación de oportunidades sociales básicas para conseguir la equidad y la justicia social y la creación de una nueva institucionalidad social que ayude a repartir el crecimiento entre los distintos sectores sociales (Sen, 2000). Así como se tiene que derrotar la creencia subyacente de que el desarrollo es una especie de lujo que solo pueden permitirse los países desarrollados, también deben sacarse lecciones de la experiencia de los países del este asiático que comenzaron su proceso de crecimiento a partir de fuertes inversiones en educación y asistencia sanitaria que mejoraron las capacidades de los habitantes.

Las metas macroeconómicas tradicionales como las del crecimiento económico estable, reducción de los costos sociales de la inestabilidad económica de corto plazo, mayor destinación de mayores recursos del PIB para inversión social, supresión del dualismo interno que divide la economía entre sectores formales e informales y la definición de las bases de una nueva institucionalidad social, requieren de un pacto social entre los distintos agentes que podría comenzar con un diálogo social como el que se plantea para el desarrollo de este Foro de Biarritz en Santiago de Chile (2007).

\section{POLIIIICAS DE EMPLEO PARA LA COHESIÓN SOCIAL}

La experiencia europea señala cómo la expansión del mercado de trabajo y la inclusión en el mismo, de sectores secularmente excluidos como las mujeres y los discapacitados, puede ser una estrategia muy eficaz contra la exclusión. El desempleo, como exclusión, se manifiesta en América Latina a través de empleos de baja calidad y escasa remuneración. Se destaca, como característica latinoamericana, la "pauperización salarial" como el mayor enemigo de la cohesión social; el eje de articulación social no puede ser la mano de obra barata como sucede hoy en la China: allí está el problema "real" de la cohesión social. ${ }^{26} \mathrm{El}$ empleo puede ser un mecanismo de inclusión e integración cuando crea nuevos frentes productivos de ocupación o de exclusión o fragmentación cuando lo hace a través del subempleo o el empleo informal. De hecho, el desempleo lleva a otras formas de exclusión como la falta de salud, la mortalidad y la pérdida de autoestima como sentido de pertenencia.

La circunstancia de que el $80 \%$ de los latinoamericanos tengan el trabajo como su principal fuente de ingreso, señala la importancia de las políticas para la ampliación y el mejoramiento de los mercados de trabajo. Con un 56\% de los empleos generados en el sector informal, el más importante desafío que tienen las políticas de empleo frente a la cohesión social es el de proteger este sector y buscar formas alternativas para su modernización. Las más recientes políticas de "flexibilización laboral”, aunque han permitido en algunos sectores avanzar hacia nuevas formas

26. PAREDES, Beatriz, Presidenta PRI de México. Discusión del documento en el evento mencionado antes. 
de producción intensiva en mano de obra frente al excesivo proteccionismo legal de antes, también han añadido una gran incertidumbre al inestable grupo de los trabajadores informales.

Distintos organismos en la región recomiendan medidas para reducir dicha incertidumbre como subsidios al desempleo, formas de compensación de pérdidas de ingresos laborales, mayores esfuerzos en el campo de la capacitación técnica y medidas contra cíclicas para protección de empleos en épocas de crisis. Mientras exista esta alta dependencia de los ingresos laborales, las posibilidades de afianzar la cohesión social serán limitadas. Una política vigorosa de estímulo social a la adquisición de activos productivos resulta clave en el camino de reducir la desigualdad social y simultáneamente, fortalecer la inclusión y la cohesión social.

\section{POLIITICAS DE PROVIIIIÓN SOCIAL DE ACTIVOS PRODUCTIVOS}

La desigualdad social está estrechamente relacionada con la falta de activos productivos en cabeza de las familias marginadas. La falta de capital, tecnología, capacitación y tierras impide a los ciudadanos de bajos niveles de ingreso superar en el corto plazo sus niveles de pobreza, forzándolos de paso a recurrir a formas de aseguramiento informal de sus riesgos mucho más costosas que las institucionales. Distintas experiencias prueban que los programas de otorgamiento de títulos de propiedad, con inversiones adicionales para adecuarlas, puede beneficiar mujeres, indígenas y afrodescendientes, como sucedió en Colombia cuando se asignaron a los afrodescendien- tes 4.6 millones de hectáreas de tierras. Algo similar sucede con el microfinanciamiento y el acceso de las mujeres al crédito, como se comprueba en los éxitos obtenidos por el Banco de Mujeres.

Planes relacionados con la provisión de microcréditos, fondos de garantías para acceder a la liquidez bancaria, programas de banca y de titulación de tierras agrarias y urbanas, proyectos de capacitación masiva y de apoyo a las microempresas, entre otras iniciativas, podrían fortalecer esta inaplazable cruzada por mejorar la participación estructural de los estratos bajos en la distribución final del ingreso productivo. Dichas políticas deben incluir la protección de los riesgos cuyo aseguramiento desborda la capacidad de los bajos niveles de ingreso. Se trata de crear mecanismos informales para compartir riesgos basados en el intercambio $^{27}$ (Holzmann, 2003) y complementarlos con políticas institucionales de prevención, atención y atenuación de riesgos a través de mecanismos como pensiones, subsidios al desempleo, redes sociales y planes de focalización que atiendan señales de mercado.

\section{POLIIIICAS De EDUCACIÓN PARA La coHESIÓN SOCIAL}

La educación y la informatización, conjugadas, contribuyen a una mayor adquisición de "activos simbólicos" propios de la nueva era global. La educación mejora la equidad, contribuye a la competitividad y es fuente de identidad en la medida en que los educandos interiorizan la valoración positiva de la igualdad de derechos y el respeto a las diferencias de género, etnia, raza, cultura propias del multiculturalismo (CEPAL, AECI, SEGIB, 2007). La educación es, además, parte medular de

27. HOLZMANN, Robert, JORGENSEN, Oteen. "Manejo social del riesgo: un nuevo marco conceptual para la protección social y más allá”, publicado en la Revista de la Facultad Nacional de Salud Pública, 2003. 
un modelo de cohesión social en la medida en que contribuye a reducir la pobreza, prepara a las personas para el ejercicio ciudadano, protege a los grupos más vulnerables y fomenta una mayor equidad en el acceso a oportunidades de bienestar (CEPAL, AECI, SEGIB, 2007). Con razón se ha dicho que identidad, multiculturalidad y equidad en las condiciones de aprendizaje son los ejes de una escuela para los nuevos tiempos (Touraine en CEPAL, AECI, SEGIB, 2007).

La situación educativa de la región que mejoró sensiblemente en las últimas décadas en materia de cobertura, muestra deficiencias preocupantes en términos de calidad; la ampliación de la educación no se ha traducido en un mejoramiento de las condiciones salariales: los jóvenes latinoamericanos están hoy mejor preparados que sus padres, pero reciben en los mismos cargos remuneraciones más bajas. Los retos educativos del futuro se deben centrar en saldar aquellas cuentas pendientes del pasado como es el caso de la alfabetización y la educación básica de jóvenes y adultos, la educación inicial, la educación especial, la educación media, la gestión educativa, la formación docente, el mejoramiento de la gestión educativa y la descentralización del sistema a través de una mayor transferencia de recursos técnicos y financieros. Asimismo, debe explorar la relación entre nuevas tecnologías de la comunicación y la educación y rescatar el concepto de la importancia de la educación pública para acometer el formidable desafío de ofrecer preparación técnica a cien millones de jóvenes latinoamericanos para producir un salto cualitativo en materia de competitividad similar al que, en su momento, dieron las economías asiáti- cas. Esta tarea demanda su articulación con el desarrollo de capacidades de innovación científicas y tecnológicas. $^{28}$

\section{POLIIIICAS De INFORMAIIZACIÓN PARA La COHESIÓN SOCIAL}

El proceso de informatización no consiste, simplemente, en entregar computadores sin redes eléctricas para iniciarlos, virtuales para conectarlos y maestros preparados para alfabetizar electrónicamente a los nuevos educandos. El PNUD, siguiendo a Castells, ha definido la informatización como la transformación histórica, multidimensional definida por la transformación de los sistemas productivo, organizativo, cultural e institucional a partir de una revolución tecnológica que no es la causa pero sí su soporte indispensable" (PNUD, 2005).

Entre las acciones puntuales que podrían conformar la nueva agenda biregional entre América Latina y Europa en este campo estarían las siguientes: acordar políticas culturales para la promoción de contenidos conjuntos; crear redes de museos, bibliotecas y archivos; acuerdos de conservación del patrimonio histórico; elaboración de archivos digitales para proteger la memoria compartida; currículum de rescate de las lenguas indígenas; programas de redes de banda ancha y desarrollo de infraestructura de las TIC; avanzar en la construcción de multiplataformas mediáticas e interconectar los centros de investigación universitaria. Es preciso no perder de vista que los procesos de informatización no consisten solamente en el avance de los computadores o aun de la tecnología informática; se requiere vincular el tema al desarrollo del conocimiento y la sociedad basada en el conocimiento. ${ }^{29}$ 


\section{polítICAS DE PARTICIPACIÓN PARA LA COHESIÓN SOCLAL}

La percepción de los latinoamericanos sobre la democracia no es optimista. De hecho, la preferencia por ésta, como sistema político, ha venido cayendo en las encuestas donde uno de cada tres ciudadanos opina que la democracia debería funcionar, pero sin parlamento ni partidos. El porcentaje de los satisfechos en América Latina con la democracia disminuyó en diez puntos porcentuales entre 1996 - 1997 y 2004 - 2005 (de $62 \%$ a $52 \%$ ) mientras que en la Unión Europea (15 países) aumentó en 8 puntos porcentuales de $48 \%$ a $56 \%$ (CEPAL, AECI, SEGIB, 2007).

La participación es la mejor estrategia para contrarrestar este preocupante desánimo democrático que se vive en la región y que puede darse a través de los mecanismos institucionales previstos en los ordenamientos legales para el ejercicio de los derechos ciudadanos, de manera espontánea frente a situaciones dramáticas como las calamidades naturales o como parte de procesos de organización social. Las políticas participativas deben, en consecuencia, abrir caminos formales para la expresión de la comunidad a través de convocatorias como referéndum, consultas e iniciativas ciudadanas que convalidan la propia democracia; canalizar el apoyo colectivo en momentos de desastres naturales a través de oficinas especializadas en su atención oportuna y especializada y promover la organización ciudadana para generar y validar, en términos de libertad y necesidad, procesos sociales inclusivos. De lo que se trata finalmente es de romper ese círculo vicioso según el cual los más excluidos socialmente son los más débiles en términos políticos (CEPAL, AECI, SEGIB, 2007).

La Convención Europea de Participación Política podría ser un excelente referente en esta tarea en la medida en que parte de la idea europea de que el problema no es la supresión del conflicto, sino el desarrollo de procesos sociales de consenso que solo son posibles a partir de una nueva conciencia social de lo individual. ${ }^{30}$ Estos procesos participativos, como serían los relacionados, por ejemplo, con la protección del consumidor, fortalecerían la institucionalidad social y la gobernabilidad. ${ }^{31}$

Una sociedad deseable no es una sociedad sin conflictos sino una sociedad con conflictos apropiadamente resueltos (Díaz, 2007). Para proporcionar marcos adecuados de desarrollo a las personas, la sociedad debe permitirles participar en las decisiones sobre cómo orientar sus principales actividades y en la discusión sobre las reglas de juego que deben regir la comunidad (SEN en Díaz, 2007) La participación colectiva en la fijación de las metas sociales y la ejecución de las políticas con la participación de sus beneficiarios legitima las normas que se expiden para hacerlas efectivas. El papel que cumplen en esta tarea actores claves como las autoridades locales es definitivo. Con razón se ha dicho que la falta de participación política empobrece la vida humana (Harrison, 2006).

\section{LASOLIDARIDAD COMO FUENTE DE COHESIÓN SOCIAL}

La globalización se ha convertido en un juego de perdedores y ganadores donde

\section{La \\ globalización se ha convertido en un juego de perdedores y ganadores donde unos países, preparados para la competencia, ganan lo que otros pierden}

30. VEUNAC, Michel, Teniente Alcalde de Biarritz. Discusión del documento en el evento mencionado antes.

31. JACIR DE LOVO, Evelyn, Delegada Presidencial para la Defensoría del Consumidor. Discusión del documento en el evento mencionado antes. 
unos países, preparados para la competencia, ganan lo que otros pierden. Se requieren políticas complementarias para evitar que esto suceda y grandes dosis de solidaridad entre países y en las regiones para que el objetivo de la equidad social vaya de la mano con el mejoramiento de las condiciones generales de productividad y competencia. En este panorama, es fundamental el papel que juegan las empresas asumiendo su responsabilidad social para apoyar las acciones afirmativas del Estado en contra de la desigualdad y de la discriminación como obstáculos que impiden la deseable meta de la cohesión social.

La solidaridad, en su acepción más sencilla, expresa la adhesión circunstancial a una causa o a la empresa de otros. Durkheim la abordó como un hecho moral y distinguió entre la solidaridad mecánica de las sociedades primitivas y la orgánica que nace en las sociedades modernas como consecuencia de la división social del trabajo. Para Parsons, la solidaridad mecánica se centra en la legitimación de las instituciones económicas y la orgánica en la de las instituciones políticas. De ésta última depende la gobernabilidad. Para los cris- tianos, en una visión algo paternalista, la solidaridad se deriva de la justicia "cuando uno de ellos toma por propias las cargas del otro y se responsabiliza junto con este de dichas cargas". La solidaridad se concreta también a través de acciones colectivas adelantadas por el Estado como las redes de solidaridad en Colombia, el Plan de Lucha contra el Hambre de Lula en Brasil o las Misiones sociales en Venezuela o por el sector privado a través de una extensa gama de actividades relacionadas con el medio ambiente, la defensa de los derechos humanos, el mejoramiento de la calidad de vida, la protección de minorías como los discapacitados o de núcleos sociales sensibles como los niños o los ancianos.

Estos casos exitosos de RSE en América Latina como los contextos dentro de los cuales se inscriben, serán examinados a profundidad en el Foro de Biarritz de Santiago (2007). Para los efectos de su relación con el tema de la gobernabilidad podemos decir que está demostrado que en aquellos países donde hay una mayor conciencia de esta solidaridad proactiva es precisamente donde está más fortalecida la institucionalidad política.

\section{BIBLIOGRAFIA}

- Almeira, Guillermo (2004). La protesta social en la Argentina (1990-2004). Buenos Aires: Ediciones Continente.

- Albert, Michel (1992) Capitalismo contra capitalismo. Barcelona, Paidós.

- Alesina, Alberto y Edward L Glaeser. (2004). Fighting Poverty in the US and Europe: A World of Difference. New York: Oxford University Press.

- Atkinson, Anthony B (1999). The Economic Consequences of Rolling Back the Welfare State, Munich Lectures in Economics. Cambridge: The MIT Press.

- - - y Joseph Stiglitz (1980). Lectures on Public Economics. Nueva York. McGraw-Hill. 
- Ayuntamiento de Barcelona (1999) Barcelona: gobierno y gestión de la ciudad. Barcelona: Ediciones Díaz de Santos.

- Banco Interamericano del Desarrollo (2000). Desarrollo más allá de la economía. Informe de Progreso Económico y Social en América Latina, Washington, D.C.

- - (2004) Inclusión Social y Desarrollo Económico en América Latina.

- Banco Mundial (2004). "Inequality in Latin America: Breaking with History", David de Ferranti y otros (comps.), Washington, D.C., World Bank Latin American and Caribbean Studies.

- - (1998). “Assessing Aid, World Bank Policy Research Report”, New York: Oxford University Press, noviembre.

- Barómetro de Gobernabilidad, (2003). Barómetro de gobernabilidad. Bogotá, Lemoine Comunicación.

- Baudin-O’Hayon, P. W. a. G. (2003). Global Governance, Transnational Organized Crime and Money Laundering. Governing Globalization. Power, Authority and Global Governance. Londres: D. Held and A. McGrew.

- Behn, R. (2001). Rethinking Democratic Accountability. Washington, D.C.: Brookings Institution.

- Birdsall, Nancy, Augusto de la Torre y Rachel Menezes (2001) Washington Contentious: Economic Policies for Social Equity in Latin America. Washington, D.C.: Carnegie Endowment for Internacional Peace and Inter-American Dialogue .

- Bobbio, Norberto (1989). Liberalismo y democracia, México: Fondo de Cultura Económica.

- Borja, Jordi (2002). “Ciudadanía y globalización”. Reforma y Democracia, No. 22, febrero.

- Bourgignon, François y Christian Morrison (2002) “The Size Distribution of Income Among World Citizens: 1820-1990". American Economic Review.

- Bowles, S. (2000). Globalization and Redistribution: Feasible egalitarianism in a competitive world. University of Massachuset Working Paper. Boston.

- Brian C. Smith (1985), Decentralization, The Territorial Dimension of The State, London: George Allen \& Unwin, 1985.

- Burki, Shahid Javed; Dillinger, William R. y Perry, Guillermo E. "Más allá del Centro: La descentralización del Estado”. Estudios del Banco Mundial sobre América Latina y el Caribe. Washington, D.C., junio de 1999.

- Bustillo, Inés y José Antonio Ocampo (2003), “Asimetrías y cooperación en el Area de Libre Comercio de las Américas", CEPAL, Serie Informes y Estudios Especiales, No. 13, mayo.

- Castells, Manuel. “La era de la información: economía, sociedad y cultura," Volumen I La Sociedad en Red. Volumen II El Poder de la Identidad. Volumen III El Fin del Milenio. España, Alianza Editorial

- Cebrián, Juan Luís (1998). La Red. Buenos Aires: Taurus-Alfaguara.

- CEPAL (1990) Transformación productiva con equidad: La tarea prioritaria del desarrollo de América Latina y el Caribe en los años noventa, Santiago de Chile.

- - (1998) El pacto fiscal: fortalezas, debilidades, desafíos. Serie Libros de la CEPAL, No 47 (LC/G.1997/Rev.1-P), Santiago de Chile, julio. 
- - (2000). Equidad, desarrollo y ciudadanía. Bogotá, D.C.: Comisión Económica para América Latina y el Caribe (CEPAL)/Alfaomega.

- - (2002). Globalización y Desarrollo. Santiago de Chile: Comisión Económica para América Latina y el Caribe.

- - (2002). Panorama Social de América Latina 2001-2002. Santiago de Chile: Comisión Económica para América Latina y el Caribe.

- - Estudio Económico de América Latina y El Caribe, Documento Informativo del Centro de Estudios para América Latina, 2003-2004.

- - Anuario Estadístico de América Latina y el Caribe 2004.

- - (2004). Panorama social de América Latina 2002-2003. Santiago de Chile.

- CEPAL, AECI, SEGIB (2007) “Cohesión Social: Inclusión y Sentido de Pertenencia en América Latina y el Caribe," enero.

- Comisión Europea. "Unión Europea, América Latina y el Caribe: Progresando juntos,” Luxemburgo, junio de 1999.

- Cornia, Giovanni Andrea (ed.) (2004).Inequality, Growth, and Poverty in an Era of Liberalization and Globalization, Oxford: Oxford University Press/United Nations University World Institute for Development Economics Research.

- Corporación Andina de Fomento. “Cultura y Economía en el Siglo XXI,” Ensayos sobre Políticas Públicas Culturales para la Región Andina, Caracas, 2005.

- Corredor, Consuelo (2004). Pobreza, Equidad, y Eficiencia Social. En Cuadernos de PNUD. MPS, Investigaciones sobre desarrollo Social en Colombia No. 1, Bogotá D. C: Panamericana.

- Deacon, Bob (2002). Tracking the Global Social Policy Discourse: From Safety Nets to Universalism, Basic Income European Network 9th Intrnational Congress Geneva Sep 12th-14th.

- De Souza Santos, Boaventura (2002). "La Globalización del Derecho. Los Nuevos Caminos de la Regulación y la Emancipación,” IILSA- Universidad Nacional de Colombia.

- Díaz, Eduardo. (2007). “Cohesión Social, Gobernabilidad y casos de Políticas Públicas de Lucha contra la Pobreza en Colombia”. Santiago de Chile.

- Diaz-Bonilla, E. and J. Babinard (2002). Globalization and Health: A Survey of Opportunities and Risks for the Poor in Developing Countries. Organización Mundial de la Salud, Documento de Trabajo de la Serie sobre Política macroeconómica y la salud. Washington.

- Domínguez, Jorge I. Y Susan Kaufman Purcell (1999), "Political Evolution in the Hemisphere", en Albert Fishlow y James Jones (eds.), The United States and the Americas: A Twenty-first Century View, The American Assembly, Columbia University, Nueva York, W.W. Norton \& Company.

- Dornbusch, Rudiger y Sebastián Edwards (1989), Economic Crises and the Macroeconomics of Populism in Latin America: Lessons from Chile and Peru, Washington, D.C., NBER.

- Echeverría, Javier (2006) "Informatización, Cultura y Sociedad del Conocimiento" Documento de debate preparado para el 7o Foro de Biarritz. 
- Epstein, Barbara, Löwy, Michael, Parritch, Leo, Petras, James y Wallerstein, Immanuel, (2005). Movimientos de Resistencia al Capitalismo Global. Barcelona: Editorial Hacer, Barcelona.

- Ffrench-Davis, Ricardo (1999). Macroeconomía, comercio y finanzas: para reformar las reformas en América Latina. Santiago: CEPAL/mcgraw-Hill Interamericana.

- Finot, Iván (2003). Descentralización en América Latina: cómo hacer viable el desarrollo local. Serie gestión pública 38. Instituto Latinoamericano y del Caribe de Planificación Económica y Social (ILPES). Dirección de Gestión del Desarrollo Local y Regional. Santiago de Chile.

- Fondo Monetario Internacional (2001). Conditionality in Fund-Supported Programs: Policy Issues. Washington: D.C., Policy Development and Review Department.

- Fundación Universitaria Politécnico Grancolombiano, Dirección Nacional de Derechos de Autor, Convenio Andrés Bello y Corporación Escenarios. "Industrias Culturales en América Latina," septiembre de 2006.

- García Canclini, Néstor (1999). La globalización imaginada. México: Buenos Aires; Barcelona; Editorial Paidós.

- - (2006). Presentación realizada en el marco del Seminario Preparatorio sobre Industrias Culturales, auspiciado por la Organización de Estados Iberoamericanos. México DF, marzo.

- Graham, C. (2002). “Crafting Sustainable Social Contracts in Latin America: Political Economy, Public Attitudes, and Social Policy”. Center on Social and Economic Dynamics Working Paper, Series \# 29.

- Graham, C. y Pettinato, Stefano (2001). Frustrated Achievers: Winners, Losers and Subjective Well Being in new Market Economies. Center on Social and Economic Dynamics. Working Papers Series No. 21, The Brookings Institution.

- Grandi, Jorge y Sschutt, Daniel. "Expectativas ante la Cumbre Unión EuropeaAmérica Latina- Caribe: Un ensayo de Aproximación," Revista Española de Desarrollo y Cooperación. No. 3, 1999.

- Grebe, Horst y otros (2005). “Cultura y Economía en el Siglo XXI,” en Ensayos sobre Políticas Públicas Culturales para la Región Andina, Corporación Andina de Fomento, Caracas.

- Harnisch, A. (2002). "Multi-Level Governance Beyond the Nation State: The End of Legitimate Democratic Politics?". The Bologna Center Journal of International Affairs, Spring.

- Harrison, Ann. "Globalization and Poverty". Working Paper, National Bureau of Economic Research, Cambridge June, 2006.

- Helleiner, Gerry (2000). "External Conditionality, Local Ownership and Development". En Transforming Development, Jim Freedman (ed.). Toronto: University of Toronto Press.

- Hobsbawm, Eric. (2003). Años interesantes una vida en el siglo XX. Buenos Aires: Edic Crítica.

- Holzmann, Robert y Jorgensen, Oteen (2003). "Manejo Social del Riesgo: un nuevo marco conceptual para la protección social y más allá,” Publicado en la Revista de la Facultad Nacional de Salud Pública. Santiago de Chile. 
- Hopenhayn, Martín (2003). "Educación, comunicación y cultura en la sociedad de la información, una perspectiva latinoamericana" CEPAL, Santiago de Chile.

- - (2005) "América Latina desigual y descentrada". Buenos Aires: Grupo Editorial Norma.

- - (2005). "Visión Filosófica de las Relaciones en la Unión Europea y América Latina," Documento presentado en el VI Foro de Biarritz en Bogotá, Colombia.

- Houllebecq, Michel (2000), "El mundo como supermercado”. Barcelona: Editorial Anagrama, Colección Argumentos.

- Huntington, Samuel (2004). Who Are We? The Challenges to America's National Identity. New York: Simon \& Schuster

- Ibarra, Pedro (2005). Manual de la Sociedad Civil y Movimientos Sociales. Barcelona: Editorial Síntesis.

- Ibáñez, Ana María, Lindert, Kathy, Woolcock, Michael (2002) "Social Capital in Guatemala: A Mixed Methods Análisis,” A Background paper for the Guatemala Poverty Assessment (GUAPA), junio.

- IRELA. "Cooperación con América Latina: El liderazgo de Europa.” Informe del Instituto de Relaciones Europeo- Latinoamericanas, 20 de abril de 1998.

- Köhler, Horst y James D. Wolfensohn (2000). “The IMF and the World Bank Group: An Enhanced Partnership for Sustainable Growth and Poverty Reduction”, Washington, D.C.

- Kuczynski, Pedro-Pablo y John Williamson (eds.) (2003). After the Washington Consensus: Restarting Growth and Reform in Latin America. Washington, D.C.: Institute for International Economics.

- Kepel, Gilles. (2005). La Revancha de Dios. Madrid: Editorial Alianza

- Lang, Jack y Le Bras, Hervé (2006). “Immigration Positive”. París: Odile Jacob, 2006.

- Latinobarómetro (2004). Informe-Resumen Latinobarómetro 2004: Una década de mediciones, www. latinobarometro.org.

- Marfán, Manuel (2005). "Rol macroeconómico de la política fiscal”, en José Antonio Ocampo (ed.), Más allá de las reformas: dinámica estructural y vulnerabilidad macroeconómica. Bogotá, CEPAL/Alfaomega, (en prensa).

- Marín, Manuel (1999). "Integración y cohesión: la experiencia europea”, documento preparado para la VI Reunión del Círculo de Montevideo, Santo Domingo, República Dominicana, 12 y 13 de noviembre.

- Monsivais, Carlos (2000). Aires de familia. Cultura y sociedad en América Latina. Barcelona: Editorial Anagrama.

- - (2006) Presentación realizada en el marco del Seminario Preparatorio sobre Industrias Culturales, auspiciado por la Organización de Estados Iberoamericanos. México DF, marzo.

- Moulian, Tomás (2000). Socialismo del siglo XXI: La quinta vía. Santiago de Chile, Editorial LOM.

- Musgrave, Richard A. (1959). The Theory of Public Finance. Tokyo: McGraw-Hill.

- Naciones Unidas (2000). Declaración del Milenio, Nueva York, Asamblea General, Cumbre del Milenio (Nueva York, 6 al 8 de septiembre). 
- - (2001). Social Dimensions of Macroeconomic Policy, Report of the Executive Committee on Economic and Social Affairs of the United Nations, New York.

- - (2002) El Consenso de Monterrey, Conferencia Internacional sobre la Financiación para el Desarrollo. Monterrey, México, marzo (www.un.org).

- Niño, Jaime Andrés (2004). Flujos Comerciales entre Europa y América Latina. En América Latina en el Comercio Global. C aracas: Corporación Andina de Fomento.

- North, Douglass C. (1990). Institutions, Institutional Change and Economic Performance, Cambridge University Press, November.

- Nussbaum. La Calidad de Vida. México, Fondo de Cultura Económica.

- Ocampo, José Antonio (2001). "Retomar la agenda del desarrollo", Revista de la CEPAL, No 74 (LC/G.2135-P), Santiago de Chile, agosto.

- - (1992), "Reforma del Estado y desarrollo económico y social en Colombia”, Análisis Político, $\mathrm{N}^{\circ} 17$, Bogotá, septiembre-diciembre.

- O’Donnell, Guillermo (2002). "Notes on the State of Democracy in Latin America”, preparado para el proyecto "The State of Democracy in Latin America”, PNUD, (mimeo).

- Palme, Joakim (2000). "Reevaluación del Estado de bienestar en los países nórdicos", en H. Muñoz (comp.). Globalización XXI: América Latina y los desafíos del nuevo milenio. Santiago: Aguilar Chilena de Ediciones.

- Persson, Torsten y Guido Tabellini (2002). "Political Economics: Explaining Economic Policy”. Zeuthen Lecture Book Series, Cambridge, Mass., The MIT Press.

- Piñón, Francisco (2006). "Los Intelectuales, Raíces y Valores Frente al Debate de la Identidad," documento de debate preparado para el VII Foro de Biarritz por la Organización de Estados Iberoamericanos, octubre.

- PNUD (1994). Informe sobre desarrollo humano, 1994, Nueva York

- - (1999). Informe sobre desarrollo humano. Nueva York.

- - (2004). La democracia en América Latina: hacia una democracia de ciudadanas y ciudadanos, Nueva York.

- - (2005) Informe Sobre Desarrollo Humano. "La cooperación internacional ante una encrucijada: Ayuda al desarrollo, comercio y seguridad en un mundo desigual”.

- - (2006) "Informe sobre Desarrollo Humano 2006: Más allá de la escasez: poder, pobreza y crisis mundial del agua," Nueva York.

- Polanyi, Karl (1957). The Great Transformation: The Political and Economic Origins of Our Time. Boston: Beacon Press.

- Poulantzas, Nicos (1970). Poder político y clases sociales en el estado capitalista. México: Siglo XXI editores.

- Quenan, Carlos. "Las Relaciones Comerciales y Financieras Europa/ América Latina y la Cumbre de Guadalajara” (presentación de Powerpoint), IHEAL, Universidad de la Sorbona, Santo Domingo, julio de 2004.

- Ramonet, Ignacio y otros (2004). Los desafíos de la globalización. México: Editores HOAC.

- Ramos, Joseph (1991). Más allá de la economía: más acá de la utopía. Santiago de Chile, CIEPLAN. 
- Rascón Banda, Víctor. Presidente de la Sociedad General de Escritores de México. Presentación realizada en el marco del Seminario Preparatorio sobre Industrias Culturales, auspiciado por la Organización de Estados Iberoamericanos. México D.F., Marzo 4, 2006.

- Real Instituto Elcano (2006). "Influencias Culturales y Sociales de las Migraciones Latinoamericanas," Documento de debate preparado para el VII Foro de Biarritz, octubre.

- Rodrik, Dani (1997). Has Globalization Gone Too Far?, Washington, D.C.: Instituto de Economía Internacional (IIE).

- - (1999). The New Global Economy and the Developing Countries: Making Openness Work, Policy Essay, No 24, Washington, D.C.: Consejo de Desarrollo de Ultramar (ODC).

- - (1999). Governing the Global Economy: does one Architectural Style fit all? Harvard University JFK School for Government Working Paper. Cambridge, MA.

- - (2001). Four Simple Principles for Democratic Governance of Globalization. Mimeo. Boston.

- - (2001) "Development Strategies for the 21st Century", in Boris Pleskovic y Nicholas Stern (eds.), Annual World Bank Conference on Development Economics 2000, The World Bank, Washington, D.C., July.

- Rojo Duque, Luis Angel. (2003). “Europa en la Economía Mundial,” Universidad Complutense de Madrid, San Lorenzo de El Escorial, 30 de junio.

- Romero de Terreros, Juan M. (2004). "Los Hispánicos en los Estados Unidos," Fundación Ortega y Gasset.

- Ros, Jaime (2000). Development Theory and the Economics of Growth, Ann Arbor, The University of Michigan Press, September.

- Rueda, Fernando. Coordinador de Programas de la Organización de Estados Iberoamericanos. Presentación realizada en el Seminario Preparatorio sobre Industrias Culturales, auspiciado por la Organización de Estados Iberoamericanos. México D.F., marzo 4, 2006.

- Sáez Vacas, F. (2004). Más allá de Internet: la Red Universal Digital. Madrid: Centro de Estudios Ramón Areces.

- Saiegh, Sebastian y Mariano Tommasi (eds.) (1998). La nueva economía política. Buenos Aires: EUDEBA.

- Samper, Ernesto (2004). El salto global: Retos de América Latina Frente a la Globalización. Madrid: Editorial Taurus.

- Sarmiento G. Alfredo. Hacia un desarrollo económico-social equitativo, participativo e incluyente. Instituto Interamericano de Desarrollo Social. Perspectivas de la política social en América Latina. Encuentro de académicos especializados en política social

- - (2007). “La Inclusión: Marco Conceptual” Documento en borrador, marzo

- Sartori, Giovanni (2001). La sociedad multiétnica. Pluralismo, multiculturalismo y extranjeros. Madrid: Ediciones Taurus.

- Sen, Amartya. (1973). On Economic Inequality. Oxford: University Press.

- - (1998) “Teorías del desarrollo a principios del siglo XXI”, en El desarrollo económico y social en los umbrales del siglo XXI, Louis Emmerij y José Núñez, (Compiladores). BID 
- - (2000). Desarrollo y Libertad. Barcelona: Editorial Planeta

- - (2002) ¿Qué impacto puede tener la Ética? Presentación en la Reunión Internacional sobre Ética y Desarrollo" del BID, en colaboración con el Gobierno de Noruega. «www.iadb.org/etica»

- Silva Lira, Iván (2005) "Desarrollo económico local y competitividad territorial”, Revista CEPAL.

- Sinn, H. W. (1997). "The Selection Principle and Market Failure in Systems Competition". Journal of Public Economics 66: 247-274.

- Sotillo, Lorenzo, José Ángel. (1998) "La Cumbre Unión Europea- América Latina y el Caribe: ¿Fin de Trayecto o Punto de Partida?”. Revista Española de Desarrollo y Cooperación. No. 3

- Stiglitz, Joseph A. (1999), “The World Bank at the Millennium”. Economic Journal 109, noviembre

- - (2002). Globalization and its Discontents. New York: W.W.Norton.

- Touraine, Alain (2005). Un Nuevo Paradigma. Barcelona: Editorial Paidós.

- UNCTAD (Conferencia de las Naciones Unidas sobre Comercio y Desarrollo) (1997), Informe sobre el comercio y el desarrollo, Ginebra. 